\title{
Toward digital validation for rapid product development based on digital twin: a framework
}

\author{
Sihan Huang ${ }^{1} \cdot$ Guoxin Wang ${ }^{1} \cdot$ Dong Lei ${ }^{2,3} \cdot$ Yan Yan $^{1}$
}

Received: 27 April 2021 / Accepted: 27 November 2021 / Published online: 1 January 2022

(c) The Author(s) 2021

\begin{abstract}
Product development should cover product design, validation, and manufacturing. In traditional product development, physical validation based on physical trial manufacturing is the key step to confirm the design scheme before physical manufacturing. However, physical validation is costly and inefficient, which could be the main obstacle to achieving rapid product development. The emergence of digital twin provides an opportunity to accelerate product development by eliminating physical validation toward digital validation in the smart manufacturing era. Therefore, a framework of rapid product development based on digital twin is proposed in this paper. During product development, the new product is designed according to the new requirements in the virtual space, in which the existing digital twins of products can be referenced. Then, an ultrahigh-fidelity virtual manufacturing system is constructed for digital trial manufacturing based on the digital twin of the manufacturing system and the design scheme of the new product. An ultrahigh-fidelity digital prototype can be obtained from digital trial manufacturing for digital validation. The new product validation is executed on the digital prototype to test its performance. The digital validation results can be used to improve the design scheme of the new product and boost the corresponding manufacturing processes. In addition, the core characteristics and key technologies of rapid product development based on digital twin are discussed. Finally, a case study is presented to implement the proposed framework and to show the effectiveness of accelerating product development.
\end{abstract}

Keywords Product development $\cdot$ Smart manufacturing $\cdot$ Digital twin $\cdot$ Digital validation

\section{Introduction}

Product development is described broadly as the transformation of a market opportunity into a product available for sale [1-3]. The fierce competition of globalization and customized demands leads to a progressively shorter time window for product development, requiring rapid responsiveness to market fluctuations [4]. The core of product development includes product design, product validation, and product manufacturing [5]. Product validation is the key step for the success of product development to evaluate the feasibility of the design scheme and find the potential error and others.

Guoxin Wang

wangguoxin@bit.edu.cn

1 School of Mechanical Engineering, Beijing Institute of Technology, Beijing 100081, China

2 University of Chinese Academy of Sciences, Beijing 100049, China

3 AVIC Technology Co. LTD, Beijing 100083, China
With the development of information technologies and advanced manufacturing technologies, the digitalization of product development is accelerating, especially in the intelligent/smart manufacturing era [6-8]. With the assistance of computer-aided design (CAD) software [9], product design can be executed in virtual space, becoming a mainstream approach in product development. Traditionally, product validation is executed on physical prototypes obtained from physical trial manufacturing to evaluate the feasibility and performance of the design scheme [10], that is, physical validation. Physical trial manufacturing is executed in a physical trial manufacturing system, resulting in a large investment cost. Besides, reiterating physical prototypes can be extremely costly as well [11]. The possibility of product validation in the virtual space was explored through computer-aided engineering (CAE) [12], digital mock-up (DMU) $[13,14]$, and virtual manufacturing/prototype [15, 16]. These technologies can boost the product development process through rapid prototyping and validation in virtual space. However, regardless of the DMU or virtual 
prototype (manufacturing), they all fairly consider realtime data in physical space. Namely, the existing validation based on DMU or virtual prototype is executed under ideal situations, resulting in validation deviations from real situations. Therefore, physical validation based on physical trial manufacturing is still required to confirm the design scheme before product manufacturing. In addition, the trial manufacturing system for physical trial manufacturing is close to but still far different from the real manufacturing system. Thus, the corresponding physical validation results could also be incorrect. In summary, the product development process is shown in Fig. 1, where gray arrows connect the product design, product validation, and product manufacturing. Product validation across two spaces (virtual space and physical space) becomes the major obstacle to rapid product development. The possibility of executing whole validation processes in the virtual space (white arrows in Fig. 1) should be investigated, in which the physical validation based on physical trial manufacturing can be eliminated to promote product development efficiency.

The emergence of digital twin $[17,18]$ provides an opportunity to remove the abovementioned obstacle [19]. The digital twin of manufacturing system [20] provides an ultrahigh-fidelity virtual manufacturing system to support the construction of digital trial manufacturing system for the trial manufacturing of new product in the virtual space. The virtual manufacturing system of digital twin is different from the abovementioned virtual manufacturing system of virtual manufacturing, which can maintain ultrahigh fidelity through seamless data transmission with the corresponding physical manufacturing system. So, it is possible to transfer from traditional costly physical trial manufacturing to digital trial manufacturing based on digital twin. The digital prototype obtained from digital trial manufacturing is closest to the real product and can achieve complete product



Fig. 1 Product developmentprocesses validation in virtual space. It will significantly accelerate product development processes. Therefore, a framework of rapid product development based on digital twin toward digital validation is proposed in this paper.

The remainder of this paper is structured as follows: Sect. 2 reviews the related works, including DMU, virtual manufacturing, and digital twin. Section 3 introduces the concept of digital twin from the perspective of product and manufacturing system. Section 4 elaborates the framework of product development based on digital twin. Section 5 discusses the core characteristics and the key technologies of the proposed digital validation framework. Section 6 presents a case study to implement the proposed framework. Section 7 concludes this paper.

\section{Related works}

There are three concepts mentioned in the introduction, namely, DMU, virtual manufacturing, and digital twin. DMU used for design validation can improve product quality and reduce development costs simultaneously [21], which is particularly important for the early stages within the design process [22]. Virtual manufacturing can be used to concurrently simulate all the activities and functions involved throughout the life cycle of a product using VR technology [23, 24], which aims to integrate diverse manufacturingrelated technologies under a common simulation environment [15]. DMU and virtual manufacturing attempted to excavate more rapid or accuracy modeling methods for virtual prototype or virtual manufacturing system. However, they are all "static" models that cannot update to the lasted state of the corresponding physical counterparts resulting in low fidelity. Therefore, a thorough review of the recent literature with a focus on using digital twins for the entire product development process will be provided in this section to highlight the gap to be filled in this paper.

The concept of digital twin was firstly proposed by Grieves at 2003 for product lifecycle management [17]. In 2010, NASA constructed the first digital twin based on probabilistic simulation to mirror the lifecycle of its flying twin [25]. Tuegel et al. [26] adopted digital twin to predict the aircraft structure life and assure its structural integrity. And then, the study of digital twin entered a period of rapid development [27, 28], especially the study on digital twin of product. Some researchers have attempted to fill the gap between product design and manufacturing based on digital twin $[29,30]$, extending the investigation and application of digital twin to manufacturing domain [31-35]. In addition, Tao et al. [36] reviewed state-of-the-art digital twins in the industry. Qi et al. [37] summarized the enabling technologies and tools for digital twin. Digital twin-driven product development synthesizes the study of product design, 
product validation, and product manufacturing based on digital twin. Lo et al. [38] reviewed product design and development based on digital twin.

Product design is the beginning of product development. As is known, digital twin is derived from the product domain $[39,40]$. Digital twin-driven product design is an indispensable piece of the digital twin landscape. Tao et al. [27] proposed a digital twin-driven product design method with the consideration of big data, which illustrates the future applications of digital twin for product design. Tao et al. [41] proposed a digital twin-driven product design framework for enabling designers to fully customize, easily compare, and better understand requirements. Sun et al. [42] developed a theoretical framework for the digital twin-driven assembly-commissioning of high precision products. Lim et al. [43] explored the engineering product family design and optimization method using digital twin. Huang et al. [44] proposed digital twin-driven reconfigurable machine tools design method to improve design accuracy and efficiency. Lin et al. [45] addressed the evolution problem of digital twin and proposed an intelligent approach for product design based on evolutionary digital twin to provide a more precise approximated model of the physical world through supervised learning.

Product validation is crucial to ensure the feasibility and effectiveness of product design. High-fidelity validation conditions based on digital twin are necessary to eliminate costly and inefficient physical validation. Schleich et al. [29] investigated the reference model of virtual products to increase its fidelity based on Skin Model Shapes. Similarly, Groen et al. [46] proposed an approach to predict the accuracy of the process chain and product shape changes in product validation based on digital twin. Digital twin-driven product validation should also address process planning problem based on product design results, which connects product design and manufacturing system to support product validation through virtual manufacturing. Liu et al. [47] proposed a digital twin-based smart process planning by reusing and evaluating process knowledge. Wang and $\mathrm{Wu}$ [48] developed a planning and scheduling system based on digital twin to analyze the management and control method for achieving uncertain workshop factors. Liu et al. [49] proposed an intelligent scheduling method for digital twin workshops considering "Feature-Process-Machine Tool" Supernetwork. Yi et al. [50] provided a digital twin reference model for smart assembly process design, which an experimental test is established to apply to reality.

Product manufacturing is the last step of product development. Product manufacturing will benefit from digital twin to increase manufacturing efficiency. Lu et al. [51] reviewed the recent development of digital twin technologies in manufacturing systems and processes, which the connotation, application scenarios, and research issues are discussed. Liu et al. [52] presented a digital twin-driven methodology for rapid individualized design of automated flow-shop manufacturing system. Digital twin-driven design of configuration, motion, control, and optimization for flowtype smart manufacturing system was studied in their further work [53]. Leng et al. [54] addressed the individualization demands problem of manufacturing system based on digital twin. Li et al. [55] studied the manufacturing-resource recommendation method for digital twin shop-floor based on manufacturing tasks semantic modeling. Besides, Wang et al. [56] discussed the digital twin data-driven smart customization resulting in a new paradigm. Wei et al. [57] proposed a consistency retention method for the digital twin of CNC machine tool between virtual space and physical space. Liu et al. [58] investigated a digital twin-based machining process monitoring method to help field operators control product quality in real time (Table 1 ).

Overall, many studies focus on digital twin-driven product development, including product design, product validation, and product manufacturing. The achievements of the digital twin make it promising to execute product validation in the virtual space completely. However, the existing literature tends to investigate the digital twin-driven product design, product validation, and product manufacturing separately. Few studies have explored product design, product validation, and product manufacturing based on digital twin

Table 1 Summary of existing literature

\begin{tabular}{llll}
\hline Reference & \multicolumn{2}{l}{ Product development based on digital twin } \\
\cline { 2 - 4 } & Product design & $\begin{array}{l}\text { Product } \\
\text { validation }\end{array}$ & $\begin{array}{l}\text { Product } \\
\text { manufacturing }\end{array}$ \\
\hline Tao et al. [27] & $\sqrt{ }$ & & \\
Tao et al. [41] & $\sqrt{ }$ & & \\
Sun et al. [42] & $\sqrt{ }$ & & \\
Lim et al. [43] & $\sqrt{ }$ & & \\
Huang et al. [44] & $\sqrt{ }$ & & \\
Lin et al. [45] & $\sqrt{ }$ & $\sqrt{ }$ & \\
Schleich et al. [29] & & $\sqrt{ }$ & \\
Groen et al. [46] & & $\sqrt{ }$ & \\
Liu et al. [47] & & $\sqrt{ }$ & $\sqrt{ }$ \\
Wang and Wu [48] & & $\sqrt{ }$ \\
Liu et al. [49] & & & $\sqrt{ }$ \\
Yi et al. [50] & & & $\sqrt{ }$ \\
Lu et al. [51] & & & $\sqrt{ }$ \\
Liu et al. [52] & & & $\sqrt{ }$ \\
Liu et al. [53] & & & \\
Leng et al. [54] & & & \\
Li et al. [55] & & & \\
Wang et al. [56] & & & \\
Wei et al. [57] & & & \\
Liu et al. [58] & & & \\
\hline
\end{tabular}


Fig. 2 Digital twin ofproduct



Physical space

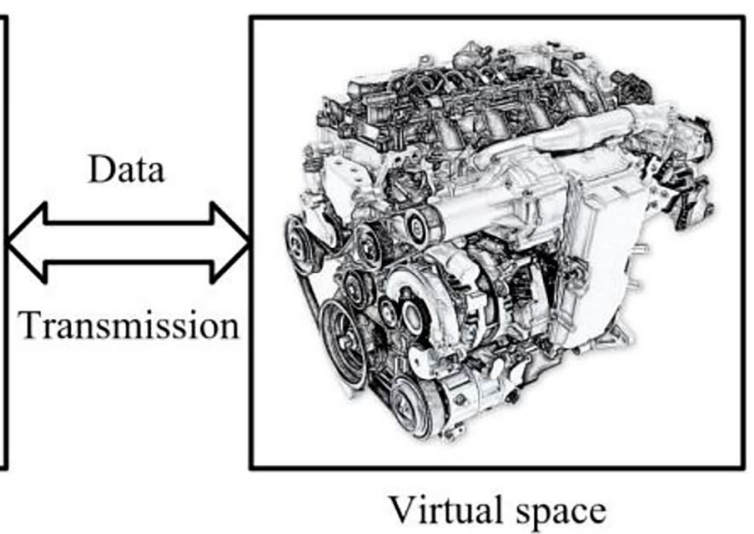

together, which is more reasonable and necessary to accelerate the speed of product development. Therefore, to fill this knowledge gap, a framework for rapid product development based on digital twin will be proposed in this paper.

\section{Digital twin of product/manufacturing system}

Although Grieves did not implicitly call it "digital twin," the concept proposed in the presentation has all the elements of digital twin: physical space, virtual space, and the link for data transmission between physical space and virtual space $[18,59]$. In addition, there are several definitions of digital twin that have been proposed by other researchers $[60,61]$.

The digital twin of product consists of three components, namely, physical product, virtual product, and the seamless data transmission between them, as shown in Fig. 2. The interaction between physical product and virtual product triggers the vitality of digital twin of product. In other words, the data flow from physical product to virtual product updates the virtual product to the latest states of the corresponding physical product to maintain its ultrahigh-fidelity characteristic, which can be used to monitor the performance of the corresponding physical product. Data feedback from virtual product to physical product can be used to support the performance optimization, maintenance scheduling, and other physical products.

Similarly, a physical manufacturing system, a virtual manufacturing system, and the data transmission between them constitute a digital twin of manufacturing system, as shown in Fig. 3. Although sharing the same basic philosophy with the digital twin of product, the construction and execution of the digital twin of manufacturing system are more complicated due to the complex core facility (machine tools) and the intricate material handling system. In addition, data acquisition and analysis are more complicated since more sensors are required resulting in tremendous data to be processed.

\section{A framework of rapid product development based on digital twin}

Product development should concern product design, product validation, and product manufacturing. Traditionally, product design is executed in virtual space; product manufacturing is executed in physical space, and product validation should be executed in both spaces. The concept of digital twin is introduced to completely support the product validation in virtual space, that is, digital validation. Therefore, a framework of rapid product development based on

Fig. 3 Digital twin of manufacturingsystem

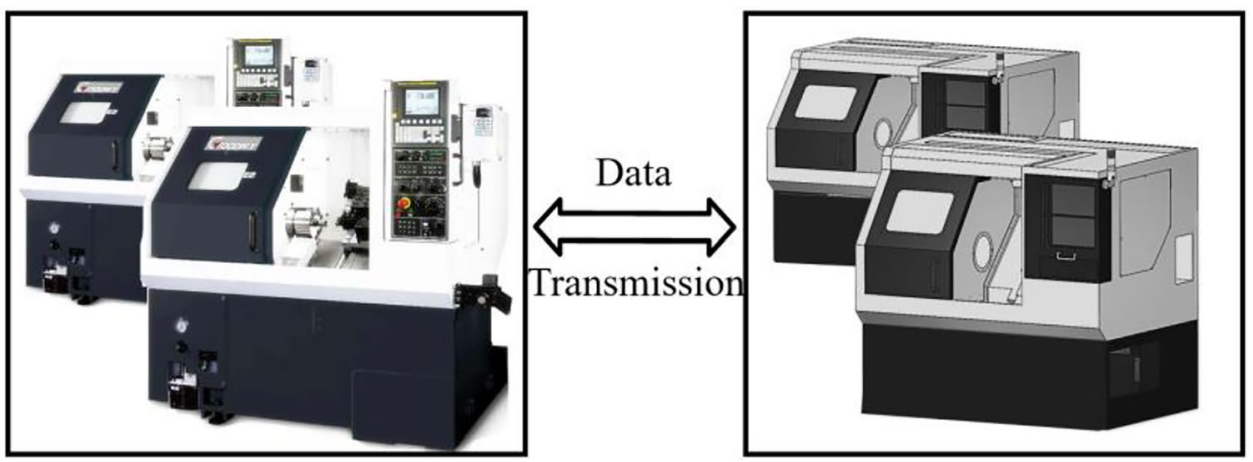


digital twin is proposed to accelerate product development processes, as shown in Fig. 4.

As the core of the rapid product development framework, the digital twin connects product design, product validation, and product manufacturing by fusing virtual space and physical space. Data flow from the physical space updates the digital twins of the manufacturing system and product in the virtual space separately, which is the foundation of completely executing product validation in the virtual space. Digital model carrying varieties of data flows among product design, product validation, and digital twin in the virtual space.

In the virtual space, the new product is designed according to the new requirements, including concept design and detail design, in which the existing digital twins of products and the design suggestions obtained from physical manufacturing processes can be referenced. Once the design process is completed, the design model of the new product can be sent to the digital trial manufacturing system for trial manufacturing in the virtual space. The digital trial manufacturing system is constructed based on the corresponding digital twin of the manufacturing system, which is joint optimization with process planning according to the design scheme of the new product. An ultrahigh-fidelity digital prototype can be obtained from digital trial manufacturing. Product validation experiments can be executed on the digital prototype to test the performance of the new product, that is, prototype validation. If prototype validation is passed, the design scheme of the new product can be used for product manufacturing; otherwise, the validation result can be used to improve the design scheme of the new product in the next iteration (Iterative Optimization).

In the physical space, physical manufacturing can be adjusted according to the manufacturing guidance from product design and product validation in the virtual space. The digital trial manufacturing system for digital validation provides the best solution to prepare the manufacturing activities of new product. Afterward, physical manufacturing of the new product begins, and the physical product can be obtained. Finally, the new product can be launched when the quality test is passed.

\subsection{Digital twin-driven product design}

The existing digital twins of products can be used to improve the design scheme of the new product. The existing digital twins of products monitor the whole lifecycle of the corresponding physical products, where the product lifecycle data flow from physical product to virtual product and the data flow from virtual product to the physical product are



Fig. 4 Rapid productdevelopment based on digital twin 
recorded. The abovementioned data is valuable for new product design. The results of diagnosis, analysis, simulation, experiments, optimization, and others on the existing digital twins of products do help to avoid the drawback of existing products during new product design. Generally, design suggestions can be obtained in the form of parameters according to the results. In addition, product design is sensitive to the market requirements. The new product should be designed to satisfy the new requirements. The new product design processes based on the digital twins of the existing products and the new requirements are presented in Fig. 5, where trade-offs could be made among them to achieve a satisfactory design scheme.

\subsection{Product validation in the virtual space based on digital twin}

\subsubsection{The construction of digital trial manufacturing system}

As mentioned before, the digital twin of manufacturing system is constructed to monitor and optimize the manufacturing activities of the developed products. Obviously, it is impossible to directly use an ultrahigh-fidelity virtual manufacturing system to execute digital trial manufacturing for new product, which will interrupt the normal operations of the digital twin of manufacturing system, resulting in unpredict disorder. In addition, the existing manufacturing system may not satisfy the manufacturing requirements of the new product. Therefore, an additional ultrahigh-fidelity virtual manufacturing system should be constructed based on the digital twin of manufacturing system for digital validation, that is, digital trial manufacturing system. The construction processes of the digital trial manufacturing system include replication and adjustment, as shown in Fig. 6. Undoubtedly, the digital trial manufacturing system should qualify for manufacturing of the new product. It is a process to map the ultrahigh-fidelity virtual manufacturing system to digital trial manufacturing system according to the design scheme of the new product. The replica of the ultrahigh-fidelity virtual manufacturing system permits the high-fidelity of the trial manufacturing system that should be as close to real manufacturing conditions as possible. So, the replica of the virtual manufacturing system should be adjusted according to the design scheme of the new product. The replica adjustment and process planning for the new product can be executed simultaneously to achieve global optimization through multiple iterations.
Fig. 5 Design process of newproduct

\section{Requirements of new product}



New product design 




Fig. 6 Constructionprocesses of digital trial manufacturing system

\subsubsection{Digital validation processes using digital trial manufacturing system}

Once the new product design and the construction of digital trial manufacturing system for the new product are completed, the design model of the new product can be sent to the digital trial manufacturing system for digital trial manufacturing. Afterward, an ultrahigh-fidelity digital prototype of the new product can be obtained. The ultrahigh-fidelity digital prototype can be used for product validation in the virtual space to verify the performance of the new design, where the potential errors or drawbacks can also be found to improve the design scheme of the new product. Digital trial manufacturing for the new product is a process that integrates the data from the digital trial manufacturing system into the raw materials according to the design scheme of the new product through manufacturing simulation. The execution processes of digital validation are shown in Fig. 7.

First, the design model of the new product is sent to the digital trial manufacturing system, where all the operations needed for manufacturing a product will be executed in the digital trial manufacturing system to "manufacture" an ultrahigh-fidelity digital prototype of the new product. Second, product validation will be executed on this ultrahigh-fidelity digital prototype (prototype validation).
Third, if necessary, the validation results are fed back to the designer for design improvement. If the design scheme is changed according to the validation results, another round of digital trial manufacturing should be executed. Obviously, the adjustments of process planning and digital trial manufacturing system are required in this digital validation round. In other words, digital validation can be executed in more than one iteration to obtain an optimal design scheme during product development. Because all activities of digital validation are executed in the virtual space, the efficiency of digital validation could be extremely high. In addition, the digital validation based on digital trial manufacturing is cost-effective, where the costly physical trial manufacturing can be eliminated.

\subsubsection{Digital validation-as-a-service}

During the entire product development process, there are many participators who play different roles, including product designer, manufacturer, and other assistant participators. Each participator type consists of one or more persons. These participators are professional in their own profession but may not be aware of the knowledge of other domains. There are communication barriers among these participators, which may disperse their attention to their own work. This situation decreases the efficiency of 
Fig. 7 Digital validationprocess



product development and countervails the advantage of digital validation as well. To make each type of participator focus on their own work and promote the product development efficiency, the concept of digital validationas-a-service (DVaaS) is proposed in this section.

Here, the digital validation based on digital twin is a service for product designer and manufacturer. Professional participators prepare the digital trial manufacturing system and other necessary elements for digital validation. Under this situation, the product designers can only focus on the design works and use the DVaaS to validate their design scheme without concerning the details of digital trial manufacturing and validation. The validation results are fed back to the designer for product improvement once the digital validation is completed. Additionally, the manufacturer will benefit from the DVaaS, which can provide the digital trial manufacturing information on the new product to assist in manufacturing preparations. The relationships among the designer, manufacturer, and DVaaS are presented in Fig. 8.

\subsection{Product manufacturing in physical space}

Once the design scheme of the new product is confirmed by digital validation, the product manufacturing in physical space begins, including physical manufacturing system adjustment, physical manufacturing, physical product, quality test, and product launch. Digital validation is an intermediate between product design and product manufacturing. On the one hand, digital validation benefits from the high-fidelity digital twin of manufacturing system, where the digital trial manufacturing system is constructed based on the ultrahigh-fidelity virtual manufacturing system that guarantees high confidence level on the validation results. On the other hand, digital validation information, including the construction and adjustment of the digital trial manufacturing system and the other manufacturing preparations, can be used to prepare the physical manufacturing system for manufacturing the new product. It will accelerate the system ramp-up [4] process for manufacturing the new product and decrease the manufacturing lead time of the new product to seize the market opportunities.

In addition, the digital trial manufacturing system for digital validation can be directly adopted as the initial ultrahigh-fidelity virtual manufacturing system to monitor the manufacturing states of the new product, which increases the construction efficiency of the corresponding digital twin of manufacturing system. Moreover, the ultrahigh-fidelity digital prototype obtained from digital trial manufacturing can be referred to when constructing the digital twin of the new product, which will increase the efficiency of constructing the digital twin of the new product.

\section{Core characteristics and key technologies of digital validation}

As mentioned in the introduction, the ultimate goal of the proposed rapid product development method based on digital twin is to eliminate the costly and inefficient physical validation during product development. The digital validation is valuable and promising for product development in the digital manufacturing era. The core characteristics and key technologies of digital validation will be discussed in this section. 
Fig. 8 Relationships amongdesigner, manufacturer, and DVaaS

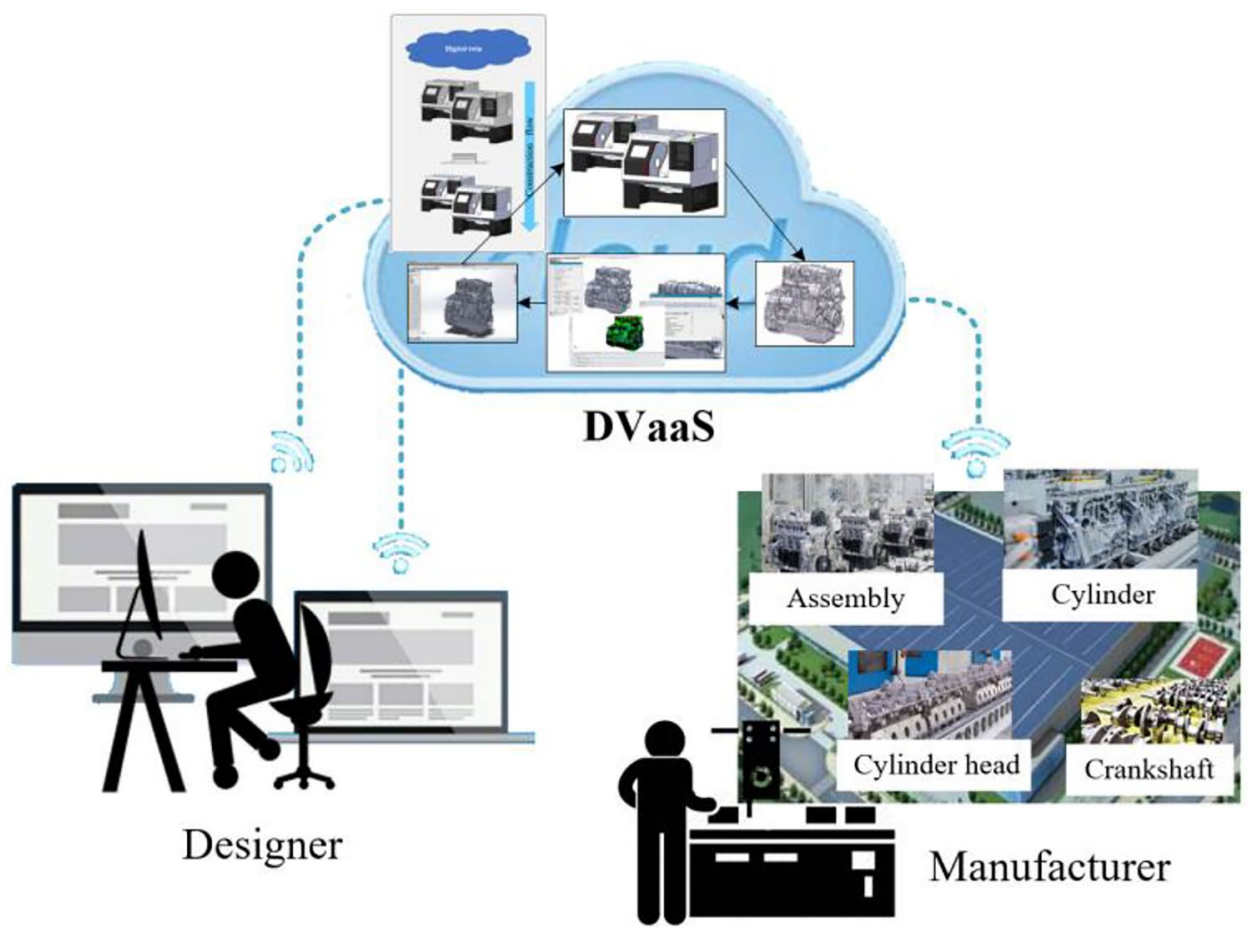

\subsection{Core characteristics of digital validation}

\subsubsection{Ultrahigh-fidelity digital trial manufacturing system}

Traditionally, the manufacturing process simulations in virtual space are executed under ideal conditions, as shown in Fig. 9a. In other words, the real-time data of the physical manufacturing process is not considered. Unlike traditional methods, the digital trial manufacturing system integrates the data of the physical manufacturing system, increasing its fidelity, as shown in Fig. 9b. In the digital twin of manufacturing system, the ultrahigh-fidelity virtual manufacturing system is continuously updated to the latest state of the corresponding physical manufacturing system through advanced information technologies. The digital trial manufacturing system is adapted from the ultrahigh-fidelity virtual manufacturing system according to the requirements of the new product, which maintains the fidelity of the ultrahigh-fidelity virtual manufacturing system as much as possible. Therefore, the digital trial manufacturing system is an ultrahigh-fidelity virtual manufacturing system for manufacturing the new product as well. An ultrahigh-fidelity digital trial manufacturing system is the foundation to eliminate the costly and inefficient physical validation.

\subsubsection{Ultrahigh-fidelity digital prototype}

The digital prototype obtained from digital trial manufacturing is different from the existing concept of DMU. The DMU is enriched by all the activities that integrate the already known knowledge into the 3D model. Direct integration processes could neglect the interactions among Multiphysics. The construction of DMU involves "adding" processes that may lose some unaware but crucial things bringing about incorrect results. In addition, the DMU
Fig. 9 (a) Traditionalmanufacturing simulation; (b) Digital twin-driven manufacturing simulation

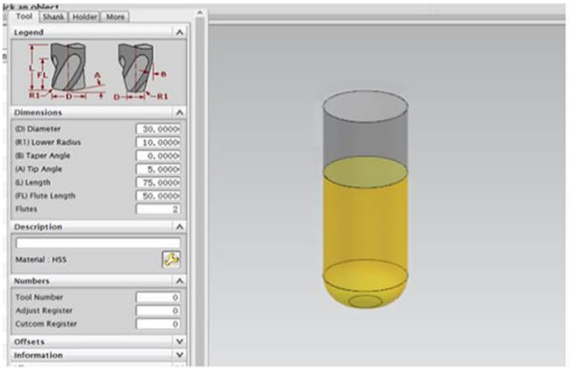

(a)

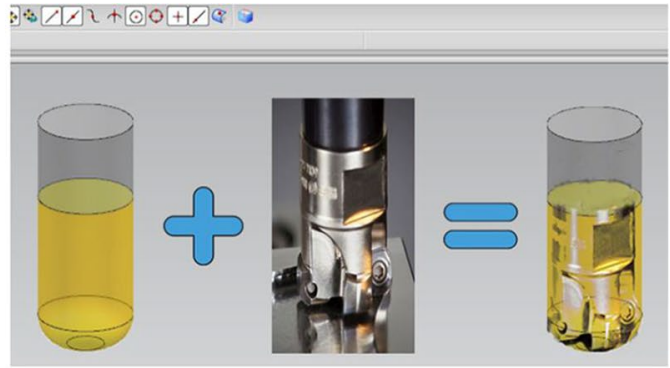

(b) 
only describes the ideal situation of product lacking data transformation between virtual space and physical space. However, the digital prototype obtained from digital trial manufacturing is "manufactured" in the ultrahigh-fidelity digital trial manufacturing system. The digital prototype not only integrates the known things but also contains the unknown stuff through the "manufacturing processes" in the virtual space. For example, the surface roughness of the digital prototype can be simulated through the manufacturing processes, as shown in Fig. 10. Definitely, the fidelity of the digital prototype is ultrahigh and much higher than that of DMU. The ultrahigh-fidelity digital prototype makes it possible to skip the product validation on the physical prototype.

\subsubsection{Digital validation as a service}

The DVaaS prepares everything needed for product trial manufacturing and validation in the virtual space (Fig. 8). It is very convenient and easy for product designer to validate the feasibility and test the performance of the design scheme of the new product through digital validation services. This process truly increases the efficiency of product design. Moreover, the elimination of costly and inefficient physical validation will considerably reduce the product development time. In addition, the reduction in manufacturing lead time benefiting from the system adjustment information of the digital trial manufacturing system from DVaaS can significantly accelerate product development.

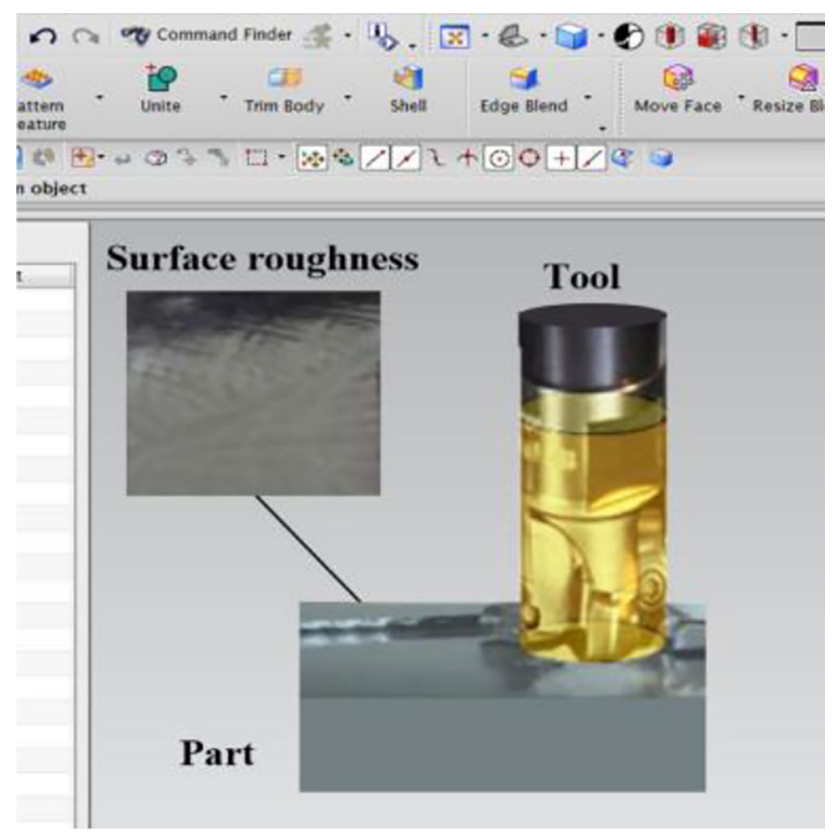

Fig. 10 Surface roughnesssimulation through "manufacturing"

\subsection{Key technologies for digital validation}

\subsubsection{Rapid adjustment of digital trial manufacturing system}

The core of digital validation is the ultrahigh-fidelity digital trial manufacturing system. Rapid adjustment of the digital trial manufacturing system is the most important aspect considering the frequent product trial manufacturing during the iterations of product design, validation, and improvement. Due to the complexity of the manufacturing system, the adjustment of the digital trial manufacturing system in a short time is difficult, in which the ultrahigh-fidelity characteristic should be maintained during system adjustment. Therefore, a changeable architecture of the digital trial manufacturing system should be adopted at the outset of construction to support the rapid adjustment.

\subsubsection{Intelligent process planning algorithm}

Process planning is a preparatory step before product trial manufacturing or product manufacturing. Considering the frequent digital trial manufacturing within the iteration of product design and validation, process planning with high efficiency and validity is crucial for successfully implementing the digital validation based on digital trial manufacturing. Intelligent algorithm combining the existing knowledge and the design information of the new product will promote the efficiency and validity of process planning for digital trial manufacturing to a higher level. Therefore, intelligent process planning algorithm embedded into digital trial manufacturing should be considered to achieve digital validation, as shown in Fig. 11.

\subsubsection{Highly effective and efficient validation method in the virtual space}

The ultrahigh-fidelity digital prototype makes it possible to verify the design scheme of the new product in the virtual space. However, product validation in the virtual space requires more effective and efficient methods compared with existing validation methods. For example, one of the most essential purposes of digital validation is to test the machining/assembly accuracy of the prototype to ensure product performance. The existing machining/assembly accuracy detection methods proposed for physical prototype are no longer suitable for the digital prototype. Therefore, the product validation method in the virtual space should be investigated thoroughly in the future. 




Fig. 11 Intelligent processplanning

\section{Case study}

Although it is too far to achieve the proposed framework based on current technical level, a prototype system is developed to tease out the implementation details of the proposed framework, as shown in Fig. 12. Also, a case study is presented in this section according to the development processes of typical rotorcraft parts, as shown in Fig. 13. The product design, product validation, and product manufacturing of the key part of rotorcraft will be elaborated in this section. The details of the digital twins of product and manufacturing system will be presented in this section as well.
There are many digital twins of the rotorcraft constructed for the operation states monitoring of the key part, as shown in Fig. 14. In the operation processes of the existing digital twin of the rotorcraft, a large quantity of data can be acquired via various sensors, especially the operation data of the key part. After data mining based on historical data from the existing digital twins, the fault reason of the key part can be diagnosed, which can be adopted to improve the design of the key part. Besides, potential issues of the existing key part could be found as well to enhance the operation security of the corresponding rotorcraft.

A manufacturing system is built for processing the key part of rotorcraft, that is, physical manufacturing system. Certainly, an ultrahigh-fidelity virtual manufacturing system is created in the virtual space according to the physical manufacturing system. The seamless data transmission between the physical manufacturing system and the ultrahigh-fidelity virtual manufacturing system connects these two parts in different spaces. Therefore, the digital twin of the manufacturing system for the key part can be obtained, which can be used to monitor the manufacturing details of the key part, as shown in Fig. 15. It is also the most fundamental basis for digital trial manufacturing of the new design of the key part.

The core of the proposed rapid product development framework involves trying to remove the product validation in physical space before manufacturing to accelerate the product development. In other words, the most important part of the proposed framework is the digital validation based on digital twin. As shown in Fig. 16, the foundation of digital validation is digital twin (bottom part of
Fig. 12 Prototype system forrapid product development based on digital twin






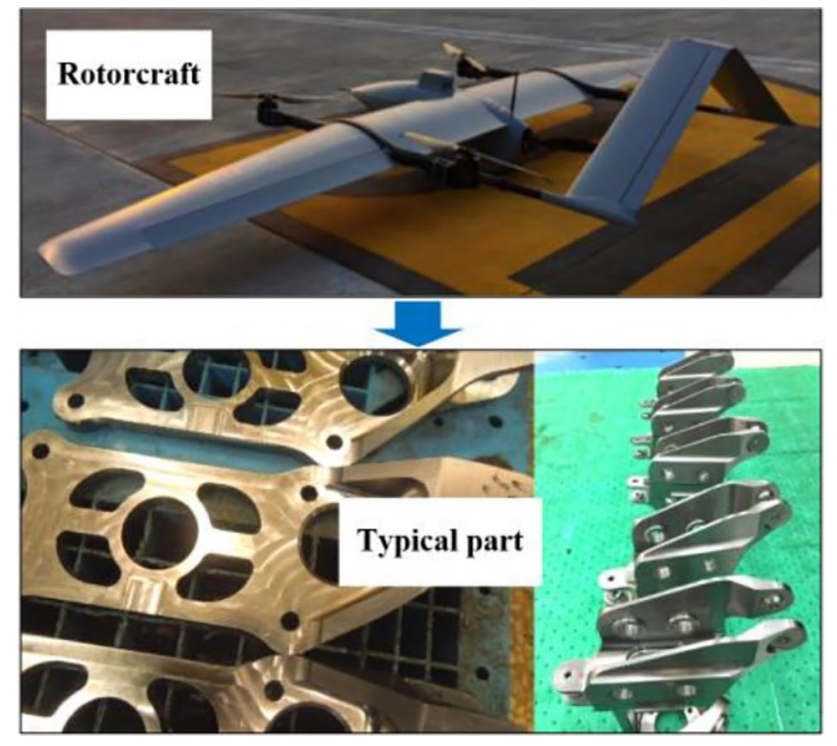

Fig. 13 A key part ofrotorcraft

Fig. 16). The digital twin of product can be referred to during the design of the new product (left middle part of Fig. 16). The digital twin of manufacturing system is the basis of the digital trial manufacturing system with ultrahigh fidelity, which will be used for the manufacturing of new product after adaptation (right middle part of Fig. 16). As shown in Fig. 16, once the new product design is complete, the ultrahigh-fidelity digital trial manufacturing system is constructed according to the digital twin of manufacturing system for the existing product, and the corresponding ultrahigh-fidelity virtual manufacturing system is replicated and adapted for the new product. The modified virtual manufacturing system (digital trial manufacturing system) is used for trial manufacturing of new product in the virtual space. Firstly, the process planning is executed automatically according to the design model of the new product and the corresponding digital trial manufacturing system (marked with red in Fig. 16), where the corresponding digital trial manufacturing system can be further adjusted to optimize the process planning and digital trial manufacturing system construction simultaneously. Secondly, the digital trial manufacturing of the new product proceeds according to the abovementioned operation sequence (marked with red in Fig. 16), which an ultrahigh-fidelity digital prototype can be obtained. Thirdly, the prototype validation is executed on the virtual space based on the "manufactured" digital prototype to test the performance of the new product in the virtual space (marked with red in Fig. 16). Finally, if the digital validation on the digital prototype of the new product is passed (marked with red in Fig. 16), the corresponding manufacturing suggestion can be adopted to accelerate the physical manufacturing processes of the new product.

In summary, the product validation of the new product in this case is executed in the virtual space completely, including process planning, trial manufacturing, prototype validation, etc. The goal of the proposed rapid product development framework is achieved through digital validation based on digital twin. The digital twin of manufacturing system provides an ultrahigh-fidelity virtual manufacturing system resulting in an ultrahigh-fidelity digital prototype for digital validation, which is really important for eliminating the costly and inefficient physical validation.

Fig. 14 The existing digitaltwins of rotorcraft

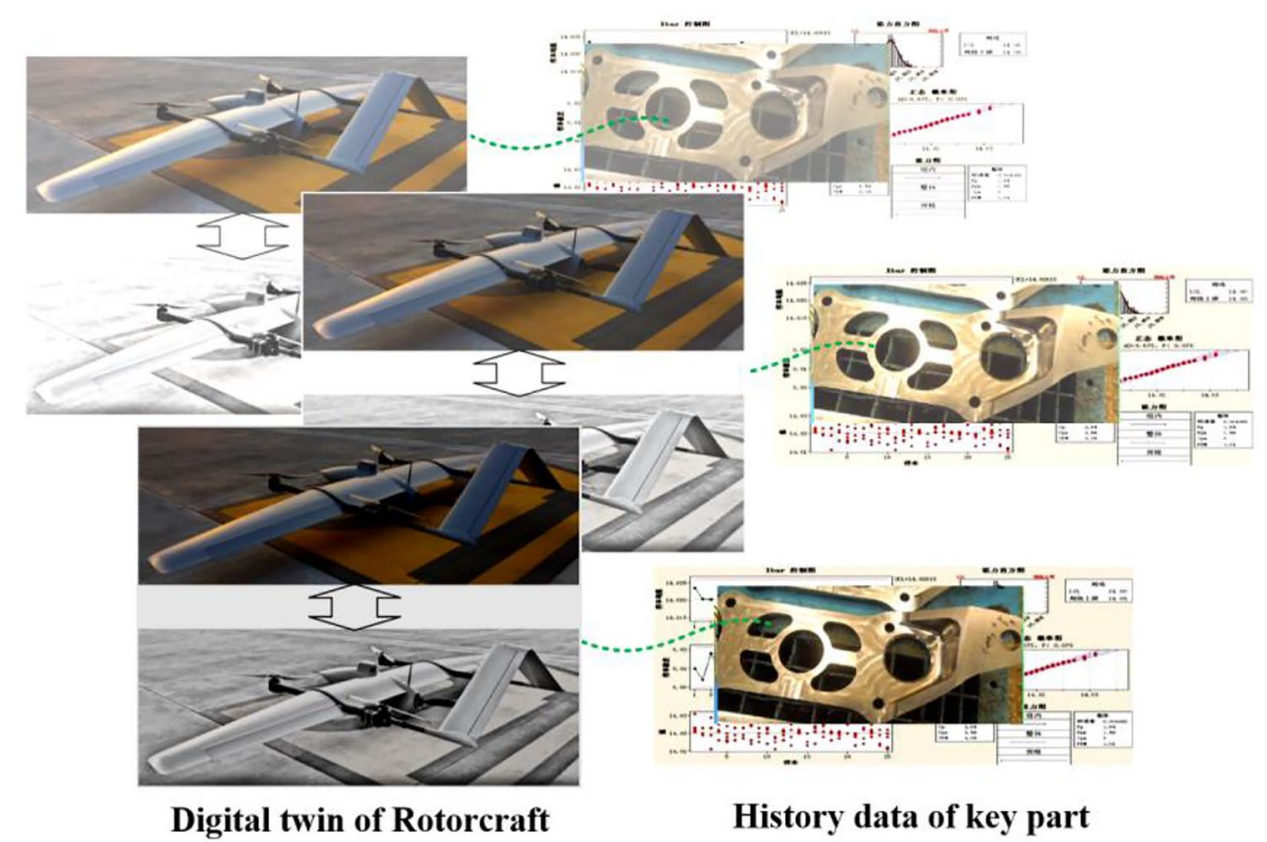


Fig. 15 Digital twin ofmanufacturing system
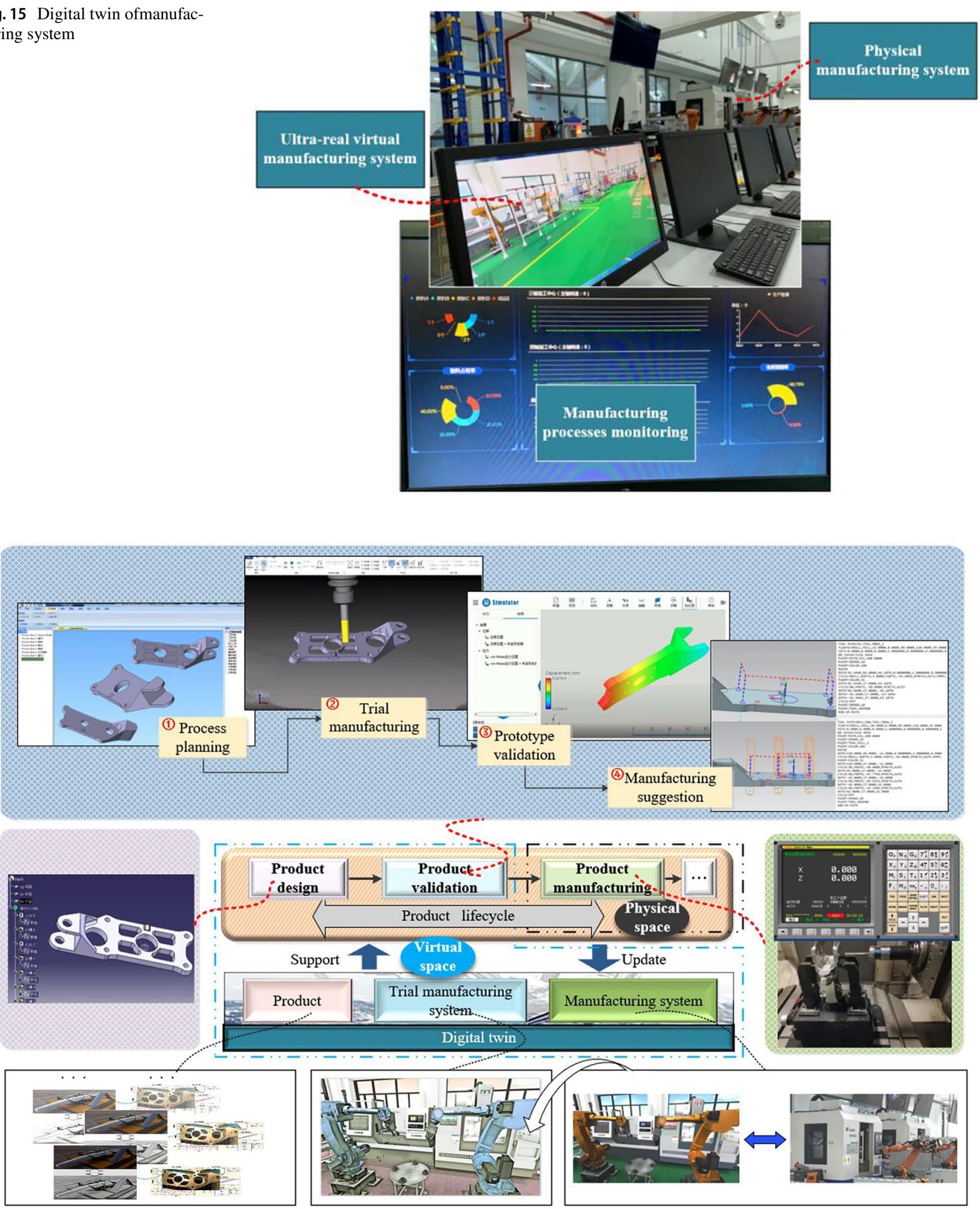

Fig. 16 Product developmentprocesses based on digital twin 


\section{Conclusion}

To achieve rapid product development, a framework of product development based on digital twin toward digital validation is proposed in this paper by compensating for the shortcomings of traditional product development. The digitalization of product design and manufacturing makes it possible to execute product validation in virtual space. The emergence of digital twin drives the product validation toward digital validation. The digital trial manufacturing system adapted from the ultrahigh-fidelity virtual manufacturing system provides a less expensive and more precise solution for product trial manufacturing in the virtual space, which can support the entire product validation in the virtual space. The ultrahigh-fidelity digital prototype obtained from digital trial manufacturing can not only be used for digital validation and design improvement, but can also accelerate the construction of the digital twin of the new product. Moreover, the results of digital validation can promote the manufacturing preparation processes of the new product as well, which will decrease the ramp-up time of new product manufacturing. The core characteristics of the proposed rapid product development framework are analyzed. The key technologies for boosting the execution of digital validation to support rapid product development are also pointed out. The case study implements the core contents of the proposed rapid product development framework, which a prototype system is developed as well.

It is very promising to eliminate costly and inefficient physical validation based on digital twin, which will significantly promote product development efficiency. However, research and development on digital twin is still accelerating, and many issues remain to be solved before the digital twin can be applied easily and smartly. Many technologies (especially the key technologies mentioned in Sect. 5.2) associated with digital twin should be further conquered in future works. Also, it is necessary to development a software platform integrating all technologies and algorithms associating with the proposed framework when the time is ripe.

Funding The China National Postdoctoral Program for Innovative Talents (BX20200053), the National Natural Science Foundation of China (51975056) and the China Postdoctoral Science Foundation (2021M700420).

Availability of data and material Not applicable.

Code availability Not applicable.

\section{Declarations}

Consent for publication This manuscript has been approved by all coauthors.

Conflict of interest The authors declare no competing interests.
Open Access This article is licensed under a Creative Commons Attribution 4.0 International License, which permits use, sharing, adaptation, distribution and reproduction in any medium or format, as long as you give appropriate credit to the original author(s) and the source, provide a link to the Creative Commons licence, and indicate if changes were made. The images or other third party material in this article are included in the article's Creative Commons licence, unless indicated otherwise in a credit line to the material. If material is not included in the article's Creative Commons licence and your intended use is not permitted by statutory regulation or exceeds the permitted use, you will need to obtain permission directly from the copyright holder. To view a copy of this licence, visit http://creativecommons.org/licenses/by/4.0/.

\section{References}

1. Takeuchi H, Nonaka I (1986) The new new product development game. Harvard Business Rev 64(1):137-146

2. Brown SL, Eisenhardt KM (1995) Product development: past research, present findings, and future directions. Acad Manage Rev 20(2):343-378

3. Hoyer WD, Chandy R, Dorotic M, Krafft M, Singh SS (2010) Consumer cocreation in new product development. J Serv Res 13(3):283-296

4. Koren Y, Heisel U, Jovan F, Moriwaki T, Pritschow G, Ulsoy G, Van Brussel H (1999) Reconfigurable manufacturing systems Cirp Ann-manuf Techn 48(2):527-540

5. Smith PG, Reinertsen DG (1998) Developing products in half the time: new rules, new tools. Van Nostrand Reinhold, New York

6. Zhou J, Li PG, Zhou YH, Wang BC, Zang JY, Liu M (2018) Toward new-generation intelligent manufacturing. Engineering 4(1):11-20

7. Lu Y, Xu X, Wang L (2020) Smart manufacturing process and system automation-a critical review of the standards and envisioned scenarios. J Manuf Syst 56:312-325

8. Guo D, Zhong RY, Lin P, Lyu Z, Rong Y, Huang GQ (2020) Digital twin-enabled Graduation Intelligent Manufacturing System for fixedposition assembly islands. Robot Comput Integr Manuf 63:101917

9. Ross DT (1960) Computer-aided design: a statement of objectives. MIT Electronic Systems Laboratory

10. Faithfull PT, Ball RJ, Jones RP (2001) An investigation into the use of hardware-in-the-loop simulation with a scaled physical prototype as an aid to design. J Eng Design 12(3):231-243

11. McHugh R, Zhang H (2011) Virtual prototyping of mechatronics for 21st century engineering and technology. Int J Eng Res Innov 3(2):69-75

12. King GS, Jones RP, Simner D (2003) A good practice model for implementation of computer-aided engineering analysis in product development. J Eng Design 14(3):315-331

13. Dai F, Reindl P (1996) Enabling digital mock up with virtual reality techniques-vision, concept, demonstrator. In Proceedings of 1996 ASME design engineering technical conference and computers in engineering (pp. 18-22)

14. Song IH, Chung SC (2009) Synthesis of the digital mock-up system for heterogeneous CAD assembly. Comput Ind 60(5):285-295

15. Shukla C, Vazquez M, Chen FF (1996) Virtual manufacturing: an overview. Comput Ind Eng 31(1-2):79-82

16. Wang GG (2002) Definition and review of virtual prototyping. J Comput Inf Sci Eng 2(3):232-236

17. Grieves M (2014) Digital twin: manufacturing excellence through virtual factory replication. White paper 1-7

18. Grieves M, Vickers J (2017) Digital twin: mitigating unpredictable, undesirable emergent behavior in complex systems. In Transdisciplinary perspectives on complex systems (pp. 85-113). Springer, Cham 
19. Morandotti D, Pelosi A (2018, July) Toward digital twin and simulation-driven new product development. RD Management Conference, Milan, Italy (pp. 1-14)

20. Tao F, Zhang M (2017) Digital twin shop-floor: a new shop-floor paradigm towards smart manufacturing. IEEE Access 5:20418-20427

21. Döllner G, Kellner P, Tegel O (2000) Digital mock-up and rapid prototyping in automotive product development. J Integr Design Process Sci 4(1):55-66

22. Schweiger W, Levichev D, Schon A, Meerkamm H (1999) Digital mock-up in the early stages of mechatronic product design. J Eng Design 10(3):235-246

23. Davidson J, Fowler J, Pantazis C, Sannino M, Walker J, Sheikhkhoshkar M, Rahimian FP (2020) Integration of VR with BIM to facilitate realtime creation of bill of quantities during the design phase: a proof of concept study. Front Eng Manag 7(3):396-403

24. Bharath VG, Rajashekar P (2015, March) Virtual manufacturing: a review. In National Conference Emerging Research Areas Mechanical Engineering Conference Proceedings (pp. 355-364)

25. Shafto M, Conroy M, Doyle R, Glaessgen E, Kemp C, LeMoigne J, Wang L (2010) Draft modeling, simulation, information technology $\&$ processing roadmap. Technology Area 11

26. Tuegel EJ, Ingraffea AR, Eason TG, Spottswood SM (2011) Reengineering aircraft structural life prediction using a digital twin. Int $\mathbf{J}$ Aerospace Eng 2011:1-14

27. Tao F, Cheng J, Qi Q, Zhang M, Zhang H, Sui F (2018) Digital twindriven product design, manufacturing and service with big data. Int J Adv Manuf Tech 94(9-12):3563-3576

28. López CEB (2021) Real-time event-based platform for the development of digital twin applications. Int J Adv Manuf Tech 1-11

29. Schleich B, Anwer N, Mathieu L, Wartzack S (2017) Shaping the digital twin for design and production engineering. CIRP Ann 66(1):141-144

30. Negri E, Fumagalli L, Macchi M (2017) A review of the roles of digital twin in cps-based production systems. Procedia Manuf 11:939-948

31. Zhuang C, Liu J, Xiong H (2018) Digital twin-based smart production management and control framework for the complex product assembly shop-floor. Int J Adv Manuf Tech 96(1-4):1149-1163

32. Park KT, Lee J, Kim HJ, Do Noh S (2020) Digital twin-based cyber physical production system architectural framework for personalized production. Int J Adv Manuf Tech 106(5):1787-1810

33. Ma J, Chen H, Zhang Y, Guo H, Ren Y, Mo R, Liu L (2020) A digital twin-driven production management system for production workshop. Int J Adv Manuf Tech 110(5):1385-1397

34. Guo H, Zhu Y, Zhang Y, Ren Y, Chen M, Zhang R (2021) A digital twin-based layout optimization method for discrete manufacturing workshop. Int J Adv Manuf Tech 112(5):1307-1318

35. Son YH, Park KT, Lee D, Jeon SW, Do Noh S (2021) Digital twinbased cyber-physical system for automotive body production lines. Int J Adv Manuf Tech 115:291-310

36. Tao F, Zhang H, Liu A, Nee AY (2018) Digital twin in industry: state-of-the-art. IEEE T Ind Inform 15(4):2405-2415

37. Qi Q, Tao F, Hu T, Anwer N, Liu A, Wei Y, Wang L, Nee AYC (2021) Enabling technologies and tools for digital twin. J Manuf Syst 58:3-21

38. Lo CK, Chen CH, Zhong RY (2021) A review of digital twin in product design and development. Adv Eng Inform 48:101297

39. Huang S, Wang G, Yan Y, Fang X (2020) Blockchain-based data management for digital twin of product. J Manuf Syst 54:361-371

40. Zhuang C, Gong J, Liu J (2021) Digital twin-based assembly data management and process traceability for complex products. J Manuf Syst 58:118-131

41. Tao F, Sui F, Liu A, Qi Q, Zhang M, Song B, Guo Z, Nee LuSCY, AY, (2019) Digital twin-driven product design framework. Int J Prod Res 57(12):3935-3953

42. Sun X, Bao J, Li J, Zhang Y, Liu S, Zhou B (2020) A digital twindriven approach for the assembly-commissioning of high precision products. Robot Comput Integr Manuf 61:101839
43. Lim KYH, Zheng P, Chen CH, Huang L (2020) A digital twinenhanced system for engineering product family design and optimization. J Manuf Syst 57:82-93

44. Huang S, Wang G, Yan Y (2020) Building blocks for digital twin of reconfigurable machine tools from design perspective. Int J Prod Res $1-15$

45. Lin TY, Jia Z, Yang C, Xiao Y, Lan S, Shi G, Zeng B, Li H (2021) Evolutionary digital twin: a new approach for intelligent industrial product development. Adv Eng Inform 47:101209

46. Groen M, Zijlstra G, San-Martin D, Post J, De Hosson JTM (2018) Product shape change by internal stresses. Mater Design 157:492-500

47. Liu Q, Zhang H, Leng J, Chen X (2019) Digital twin-driven rapid individualised designing of automated flow-shop manufacturing system. Int J Prod Res 57(12):3903-3919

48. Wang Y, Wu Z (2020) Model construction of planning and scheduling system based on digital twin. Int $\mathbf{J}$ Adv Manuf Tech 109(7):2189-2203

49. Liu Z, Chen W, Zhang C, Yang C, Cheng Q (2021) Intelligent scheduling of a feature-process-machine tool supernetwork based on digital twin workshop. J Manuf Syst 58:157-167

50. Yi Y, Yan Y, Liu X, Ni Z, Feng J, Liu J (2021) Digital twin-based smart assembly process design and application framework for complex products and its case study. J Manuf Syst 58:94-107

51. Lu Y, Liu C, Kevin I, Wang K, Huang H, Xu X (2020) Digital Twindriven smart manufacturing: connotation, reference model, applications and research issues. Robot Comput Integr Manuf 61:101837

52. Liu J, Zhou H, Tian G, Liu X, Jing X (2019) Digital twin-based process reuse and evaluation approach for smart process planning. Int J Adv Manuf Tech 100(5-8):1619-1634

53. Liu Q, Leng J, Yan D, Zhang D, Wei L, Yu A, Zhao R, Zhang H, Chen X (2021) Digital twin-based designing of the configuration, motion, control, and optimization model of a flow-type smart manufacturing system. J Manuf Syst 58:52-64

54. Leng J, Liu Q, Ye S, Jing J, Wang Y, Zhang C, Zhang D, Chen X (2020) Digital twin-driven rapid reconfiguration of the automated manufacturing system via an open architecture model. Robot Comput Integr Manuf 63:101895

55. Li X, Wang L, Zhu C, Liu Z (2021) Framework for manufacturingtasks semantic modelling and manufacturing-resource recommendation for digital twin shop-floor. J Manuf Syst 58:281-292

56. Wang X, Wang Y, Tao F, Liu A (2021) New paradigm of data-driven smart customisation through digital twin. J Manuf Syst 58:270-280

57. Wei Y, Hu T, Zhou T, Ye Y, Luo W (2021) Consistency retention method for CNC machine tool digital twin model. J Manuf Syst 58:313-322

58. Liu S, Lu S, Li J, Sun X, Lu Y, Bao J (2021) Machining processoriented monitoring method based on digital twin via augmented reality. Int J Adv Manuf Tech 113(11):3491-3508

59. Jiang H, Qin S, Fu J, Zhang J, Ding G (2021) How to model and implement connections between physical and virtual models for digital twin application. J Manuf Syst 58:36-51

60. Glaessgen E, Stargel D (2012, April) The digital twin paradigm for future NASA and US Air Force vehicles. In 53rd AIAA/ASME/ ASCE/AHS/ASC Structures, Structural Dynamics and Materials Conference 20th AIAA/ASME/AHS Adaptive Structures Conference 14th AIAA (p. 1818)

61. Reifsnider K, Majumdar P (2013) Multiphysics stimulated simulation digital twin methods for fleet management. In 54th AIAA/ ASME/ASCE/AHS/ASC Structures, Structural Dynamics, and Materials Conference (p. 1578)

Publisher's Note Springer Nature remains neutral with regard to jurisdictional claims in published maps and institutional affiliations. 